EPJ Web of Conferences 44, 03004 (2013)

DOI: $10.1051 /$ epjconf/20134403004

(C) Owned by the authors, published by EDP Sciences, 2013

\title{
First principal studya of structural, electronic and thermodynamic properties of KTaO3-perovskite
}

\author{
H.Bouafia ${ }^{1, *}$, A.Akriche ${ }^{1}$, R.Ascri ${ }^{1}$, L.Ghalouci ${ }^{1}$, B.Sahli ${ }^{4}$, S.Hiadsi ${ }^{1}$, B.Abidri ${ }^{2}$, B.Amrani ${ }^{3}$ \\ 1-Laboratoire de Microscope Electronique et Sciences des Matériaux, département de physique, USTO. BP1505 \\ El m'naouar, Oran 31000, Algérie. \\ 2-Laboratoire des Matériaux Magnétiques, Université Djillali Liabès, Sidi Bel-Abbes 22000, Algérie. \\ 3-Département de Physique; Université d'Oran es-senia, Algérie. \\ 4-Engineering Physics Laboratory, University Research of Tiaret14000, Algeria.
}

\begin{abstract}
The results of first-principles theoretical study of structural, elastic, electronic and thermodynamic properties of $\mathrm{KTaO} 3$ compound, have been performed using the full-potential linear augmented plane-wave method plus local orbitals (FP-APW+lo) as implemented in the Wien $2 \mathrm{k}$ code. The exchange-correlation energy, is treated in generalized gradient approximation (GGA) using the Perdew-Burke-Ernzerhof (PBE96) and PBEsol, Perdew 2008 parameterization. Also we have used the Engel-Vosko GGA formalism, which optimizes the corresponding potential for band structure calculations. The calculated equilibrium parameter is in good agreement with other works. The elastic constants were calculated by using the Mehl method. The electronic band structure of this compound has been calculated using the Angel-Vosko (EV) generalized gradient approximation (GGA) for the exchange correlation potential. We deduced that $\mathrm{KTaO} 3$-perovskite exhibit an indirect from $\mathrm{R}$ to $\Gamma$ point. To complete the fundamental characterization of $\mathrm{KTaO} 3$ material we have analyzed the thermodynamic properties using the quasi-harmonic Debye model.
\end{abstract}

Keywords: DFT, ab initio calculations, elastic properties, Debye model, thermodynamic properties.

\section{INTRODUCTION}

Materials that adopt perovskite structure are of a great interest because of their electrical properties, magnetic and optical behavior. These properties are sensitive to temperature, pressure and phase changes. The perovskite structure of higher symmetry is a structure of cubic symmetry and its space group is $F m \overline{3} m$. Many $\mathrm{ABO}_{3}$ compounds like KTaO3have a perovskite structure with cubic symmetry at room temperature [1]. It is well known that $\mathrm{KTaO}_{3}$ undergoes no ferroelectric phase transition like other perovskites . Therefore, this cubic perovskite has been the object of many far- infrared reflectivity (IR) and Raman studies as its lowest longwavelength optical phonon softens with decreasing temperature.

\section{METHOD OF CALCULATION}

In this paper, the full potentiel-linearized augmented plane wave plus local orbital (FPLAPW)+lo approach has been used to investigated structural, elastic, electronic and thermodynamic properties of $\mathrm{KTaO}_{3}$-perovskite within the framework of the density functional theory (DFT) [2] as implemented in the Wien2K code [3]. The GGA approximation [4] has been employed for exchange-correlation potential to calculated

*hamza.tssmegmaill.com 
structural properties of $\mathrm{KTaO}_{3}$ compound. Concerning the electronic properties, we have used the EV GGA [5] approximation which describes much better the latter properties. We expand the basis function up to $\mathrm{R}_{\mathrm{MT}} \cdot \mathrm{K}_{\max }=8.5$, where $\mathrm{R}_{\mathrm{MT}}$ is the plane wave radii and $\mathrm{K}_{\max }$ is the maximum modulus for reciprocal lattice vectors. The maximum value for partial waves inside atomic spheres is $1_{\max }=10$. The $\mathrm{k}$ integration over the Brillouin zone is performed up to a $(10,10,10)$ grid in the irreducible Brillouin zone [6]. The muffin-tin radii of $\mathrm{KTaO}_{3}$ compound chosen in our calculation are $1.9,1.7$, and 1.6 for $\mathrm{K}$, Ta, and $\mathrm{O}$ respectively.

\section{RESULTS AND DISCUSSIONS 3.1.STRUCTURAL AND ELASTIC PROPERTIES}

We have calculated structural parameters using both FP-LAPW. The total energy is obtained as a function of lattice parameters and fitted to the Murnaghan equation of state to obtain equilibrium lattice constant $(\mathrm{a})$, bulk modulus $\left(\mathrm{B}_{0}\right)$, and its pressure derivative (B'). We present, in Fig. 1, structural optimization curves obtained by using the FP-LAPW method. We report, in Table 1, our calculated values along with results of other theoretical and experimental works.

For our compounds, the equilibrium lattice constant is overestimated than the experimental value as is evident with the use of GGA method. Ours calculated results are similar with the experimental [8,9] and theoretical study [7].

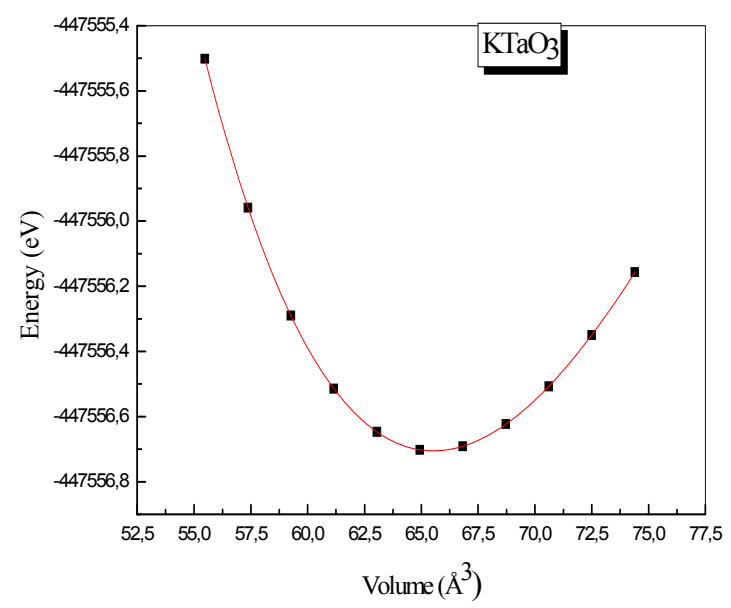

Fig 1 : Total energy versus volume curve for $\mathrm{KTaO}_{3}$.

The elastic properties define the properties of material undergoes stress, mechanical deformation, and then its returns to its original shape after stress ceases. These properties play an important part in providing valuable information about the binding characteristic between adjacent atomic planes, anisotropic character of binding and * hamza.tssmegmaill. com structural stability. Hence, to study the stability of this compound in perovskite structure, we have calculated the elastic constants at equilibrium lattice parameter. The elastic moduli require knowledge of the derivative of the energy as a function of the lattice strain. It is possible to choose this strain in such a way that the volume of the unit cell is preserved. In the case of cubic system, there are three independent elastic constants, named, $\mathrm{C}_{11}, \mathrm{C}_{12}$, and $\mathrm{C}_{44}$. Thus for their calculation, we have used the Mehl method [10].

To calculate the coefficients $\mathrm{C}_{11}$ and $\mathrm{C}_{12}$, we have used the volume-conserving orthorhombic strain tensor [11].

$\vec{\varepsilon}=\left(\begin{array}{ccc}\delta & 0 & 0 \\ 0 & -\delta & 0 \\ 0 & 0 & \frac{\delta^{2}}{\left(1-\delta^{2}\right)}\end{array}\right)$

The application of this strain changes the total energy from its unstrained value to:

$\mathrm{E}(\delta)=\mathrm{E}(0)+\left(\mathrm{C}_{11}-\mathrm{C}_{12}\right) \mathrm{V} \delta^{2}$

Where $\mathrm{E}(0)$ is the energy of the unstrained lattice at the equilibrium volume.

For the calculation of the elastic constant $C_{44}$, we used the volume-conserving monoclinic strain tensor:

$\vec{\varepsilon}=\left(\begin{array}{ccc}0 & \delta / 2 & 0 \\ \delta / 2 & 0 & 0 \\ 0 & 0 & \delta^{2} /\left(1-\delta^{2}\right)\end{array}\right)$

This changes the total energy to:

$\mathrm{E}(\delta)=\mathrm{E}(0)+1 / 2\left(\mathrm{C}_{44}\right) \mathrm{V} \delta^{2}$.

In the present study, $\delta=0.01,0.03$ and 0.05 are applied for all the cases.

The traditional mechanical stability conditions $(P=$ $0 \mathrm{GPa})$ in cubic crystals on the elastic constants are known as: $\mathrm{C} 11-\mathrm{C} 12>0, \mathrm{C} 11>0, \mathrm{C} 44>0, \mathrm{C} 11+$ $2 \mathrm{C} 12>0, \mathrm{C} 12<\mathrm{B}<\mathrm{C} 11$.

Table 1. Calculated lattice parameter $a(\AA)$, the cohesive energy (eV/cell), bulk modulus $B_{0}(\mathrm{GPa})$ and its pressure derivatives $\mathrm{B}^{\prime}$ and the elastic constants $\mathrm{C}_{11}, \mathrm{C}_{12}, \mathrm{C}_{44}(\mathrm{GPa})$ of $\mathrm{KTaO}_{3}$ compared to some experimental and other theoretical works.

\begin{tabular}{|c|c|c|}
\hline \multirow{4}{*}{$\mathrm{a}_{0}$} & Present work: GGA(PBE96) & 4.042 \\
\hline & Present work: GGA(PBEsol, Perdew 2008) & 3.991 \\
\hline & Experiment & $3.988[8]$ \\
\hline & Other works & $3.950[7]$ \\
\hline \multirow{4}{*}{$\mathrm{B}_{0}$} & Present work: GGA(PBE96) & 183.5106 \\
\hline & Present work: GGA(PBEsol, Perdew 2008) & 198.9307 \\
\hline & Experiment & $218[9]$ \\
\hline & Other works & $224.85[7]$ \\
\hline \multirow{4}{*}{$\mathrm{B}^{\prime}$} & Present work: GGA(PBE96) & 4.364 \\
\hline & Present work: GGA(PBEsol, Perdew 2008) & 4.3754 \\
\hline & Experiment & - \\
\hline & Other works & $3.695[7]$ \\
\hline \multirow{4}{*}{$\mathrm{E}_{\mathrm{coh}}$} & Present work: GGA(PBE96) & 34.231 \\
\hline & Present work: GGA(PBEsol, Perdew 2008) & 35.969 \\
\hline & Experiment & - \\
\hline & Other works & - \\
\hline
\end{tabular}




\begin{tabular}{|c|c|c|}
\hline & Present work: GGA(PBE96) & 422.113 \\
$\mathrm{C}_{11}$ & Experiment & $431[8]$ \\
& Other works & 440.75 \\
& {$[7]$} \\
\hline & Present work: GGA(PBE96) & 64.209 \\
$\mathrm{C}_{12}$ & Experiment & $103[8]$ \\
& Other works & $65.58[7]$ \\
\hline \multirow{3}{*}{$\mathrm{C}_{44}$} & Present work: GGA(PBE96) & 169.27 \\
& Experiment & $109[8]$ \\
& Other works & $85.55[7]$ \\
\hline
\end{tabular}

\subsection{ELECTRONIC PROPERTIES}

The calculated electronic band structure for $\mathrm{KTaO}_{3}$ along high-symmetry directions in the BZ and total (TDOS) densities of states are shown in Fig.2., where symmetry points $\Gamma(0,0,0), \mathrm{X}(1,0$, $0), M(1,1,0)$ and $R(1,1,1)$ are indicated in units of $\pi / \mathrm{a}$ along with the symmetry axes: $\Delta(\mathrm{x}, 0,0), \mathrm{Z}(1$, $\mathrm{x}, 0), \Sigma(\mathrm{x}, \mathrm{x}, 0)$ and $\Lambda(\mathrm{x}, \mathrm{x}, \mathrm{x}), \mathrm{x}$ being in the range $0<x<1$. We found that they have an indirect band gap with the maximum of the valence band lying at the R-point and the minimum of the conduction band lying at the $\Gamma$-point. It is well known that the GGA usually underestimate the energy gap $[13,14$, 15]. The important features of the band structure (main band gaps and valence band widths) and a comparison of our results with the experimental and other theoretical data are given in Table.2. Our calculated energy gaps are about $42 \%$ smaller than the experimental ones for the GGA (PBE96) ,43\% for the GGA (PBEsol, Perdew 2008) and $30 \%$ for the GGA-EV. Our results for the valence band widths are similar to those found experimentally.

The bands between $-17,45$ and $-15,72 \mathrm{eV}$ are mainly the contribution of $O 2 s$, Ta $6 s$ and Ta $5 d$, the second region below the Fermi level is between $-11,19$ and $-10,65 \mathrm{eV}$ is only the contribution of $K 3 p$, The valence bands lying between $-5,51 \mathrm{eV}$ and the Fermi level are mainly due to $\mathrm{O} 2 p$ states hybridized with Ta $5 d$, which means the existence of a covalent type bond between the $\mathrm{O}$ and the Ta.

We calculated the total valence charge densities in the [110] direction as show in Fig 3. The charge occurs from the Ta atoms to $\mathrm{O}$ atoms because the latter is more electronegative. While, the $\mathrm{K}-\mathrm{O}$ band is characterized by covalent bond character.
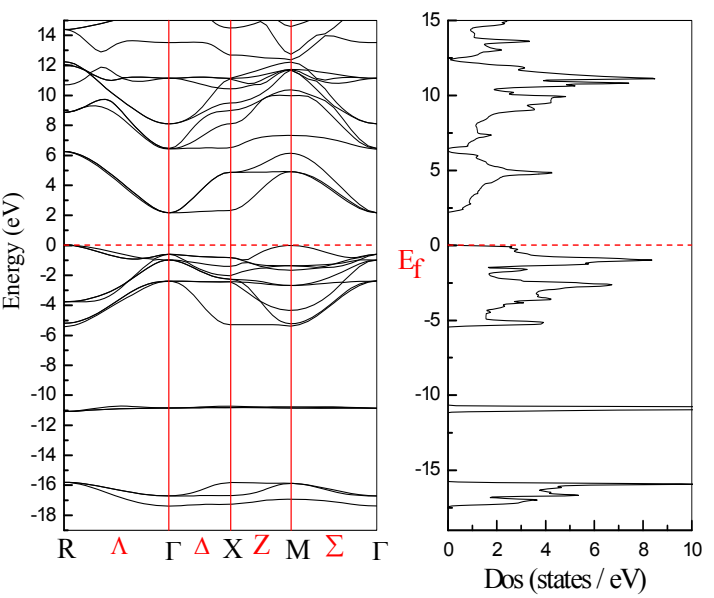

Fig 2 : The total density of states and the band structure for $\mathrm{KTaO}_{3}$.

Table 2: Calculated bandgap and the valence band widths of $\mathrm{KTaO} 3$.

\begin{tabular}{|c|c|c|c|}
\hline \multirow{5}{*}{$\mathrm{Eg}(\mathrm{eV})$} & \multirow{3}{*}{$\begin{array}{c}\text { Present } \\
\text { work }\end{array}$} & GGA(PBE96) & 2.151 \\
\hline & & GGA(PBEsol, Perdew 2008) & 2.133 \\
\hline & & GGA-EV & 2.6164 \\
\hline & \multicolumn{2}{|r|}{ Experiment } & $\begin{array}{l}3.75[17] \\
3.42[18] \\
\end{array}$ \\
\hline & \multicolumn{2}{|r|}{ Other works (LDA) } & $2.158[7]$ \\
\hline \multirow{5}{*}{ UVBW } & \multirow{3}{*}{$\begin{array}{c}\text { Present } \\
\text { work }\end{array}$} & GGA & 5.4375 \\
\hline & & GGA(PBEsol, Perdew 2008) & 5.5566 \\
\hline & & GGA-EV & 5.1358 \\
\hline & \multicolumn{2}{|r|}{ Experiment } & $5.5[16]$ \\
\hline & \multicolumn{2}{|r|}{ Other works (LDA) } & $5.637[7]$ \\
\hline
\end{tabular}

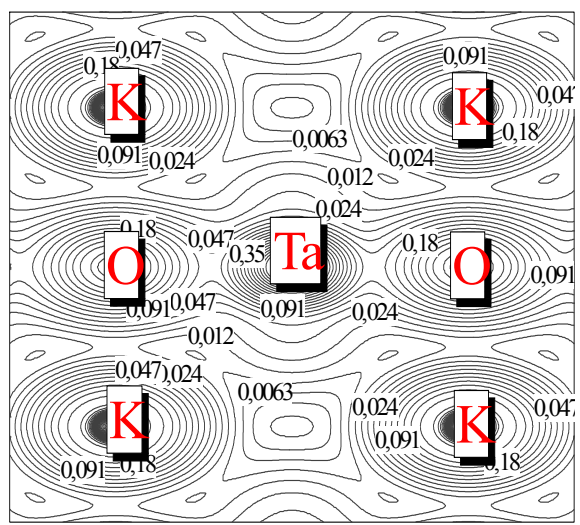

Fig.3. Calculated charge density along the [110] direction of $\mathrm{KTaO}_{3}$. 


\subsection{THERMODYNAMIC PROPERTIES}

To investigate the thermodynamic properties of $\mathrm{KTaO}_{3}$, we apply the quasi-harmonic Debye model [19], The thermal properties are monitored in the temperature range from 0 to $500 \mathrm{~K}$ at various pressures from 0 to $10 \mathrm{GPa}$, where the quasiharmonic model is probably valid, since we are far from the melting temperature. Temperature and pressure effects on the cell volume are shown in Fig.4 .At a fixed pressure, the volume increases monotonically with temperature, but the rate of increase is very moderate. On the other hand, at affixed temperature, the volume decreases when the pressure augments.

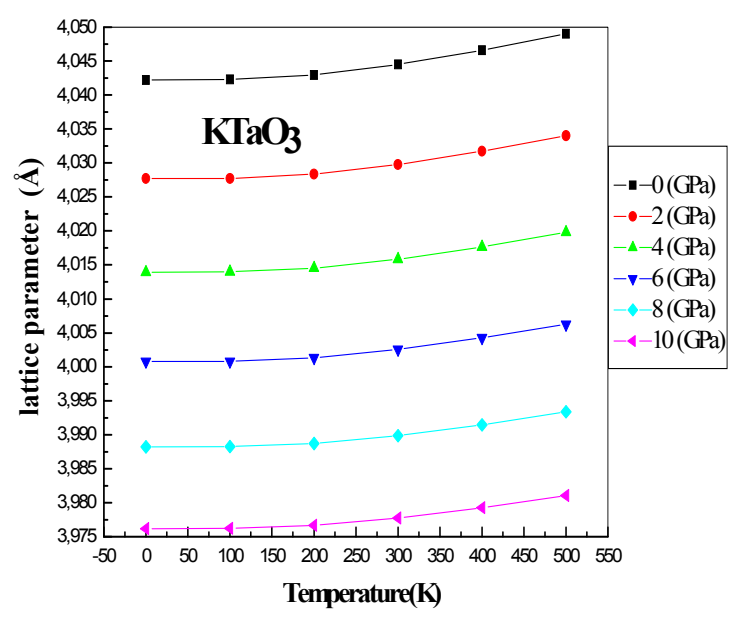

Fig 4: Variation of lattice parameter as function of temperature for $\mathrm{KTaO}_{3}$ at different pressures.

In Fig.5, we present the evolution of bulk modulus as function of temperature in the $0-500 \mathrm{~K}$ range at various pressures from 0 to $10 \mathrm{GPa}$. The shape of the curve is nearly linear. The increased of bulk modulus following the increase in pressure at given temperature. The results are due to the fact the effect of increasing pressure on material is similar as decreasing temperature of material. It is clear that the increase in temperature on material causes a significant reduction of its hardness.

*hamza.tssm@gmaill.com

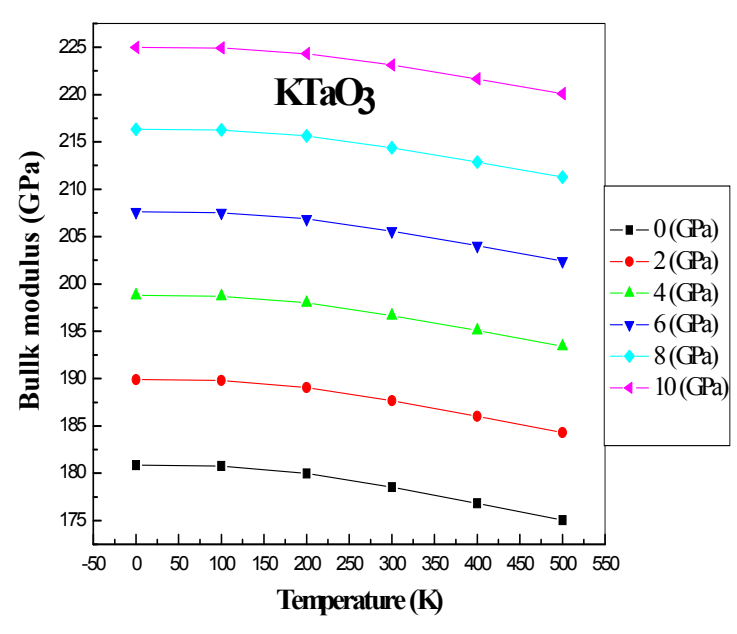

Fig 5: Variation of the bulk modulus versus temperature at various pressures for $\mathrm{KTaO}_{3}$.

\section{CONCLUSIONS}

The structural, elastic, electronic and thermodynamic properties are investigated using (FP-LAPW)+lo approach based on densityfunctional theory. The exchange- correlation potential was calculated with the frame of generalized gradient approximation (GGA) and (EV -GGA).Our total energy calculations for groundstate show that $\mathrm{KTaO}_{3}$ compound adopt perovskite structure. The calculated lattice parameter is in good agreement with the experimental and theoretical reports. The bulk modulus and its pressure derivative were predicted. All elastic constants calculated obey to stability criteria. The partial contribution from each atom to the total density of states was calculated. From the band structure, $\mathrm{KTaO}_{3}$-perovskite exhibits an indirect from $\mathrm{R}$ to $\Gamma$. point. Finally, we have conducted a detail analysis of thermodynamic properties using the quasi-harmonic Debye-model.

\section{References}

[1] M. E. Lines and A. Glass, Principles and Applications of Ferroelectric and Related Materials (Clarendon Press, Oxford, 1977).

[2] P. Hohenberg, W. Kohn, Phys. Rev. 136 (1964) B864.

[3] P. Blaha, K. Schwarz, P. Sorantin, and S.K. Trickey, Comput. Phys. Commun. 59 (1990)339.

[4] Z. Wu, R.E. Cohen, Phys. Rev. B 73 (2006) 235116.

[5] E. Engel and S.H. Vosko, Phys. Rev. B 47 (1993) 13164. 
[6] P. Blochl, O. Jepsen, and O. K. Andersen, Phys. Rev. B 49 (1994) 16223.

[7] Suleyman Cabuk Phys. Status Solidi B, Vol.247, No.1, p. 93-97, ( 2010 )

[8] Y. Shiozaki, E. Nakamura, and T. Mitsui (eds.), Ferroelectrics and Related Substances. Landolt-Börnstein: Numerical Data and Functional Relationships in Science and Technology, Vol. 36: Oxides (SpringerVerlag, Berlin, 2002).

[9] R. Comes and G. Shirane, Phys. Rev. B 5, 1886 (1972).

[12] Our calculated elastic constants are summarized in Table 1, they obey these stability conditions.

[10] M. J. Mehl, Phys. Rev. B 47, (1993) 2493.

[11] M.J. Mehl, J.E. Osburn, D.A. Papaconstantopoulos and B.M. Klein, Phys. Rev. B 41 (1990) 10311-10323.
[12] D. C. Wallace, Thermodynamics of Crystals, Willey, New York; (1972).

[13] P. Dufek, P. Blaha, K. Schwarz, Phys. Rev. B 50 (1994) 7279.

[14] E. Engel, S. H.Vosko, Phys. Rev. B 47 (1993) 13164.

[15] S. Fahy, K. J. Chang, S. G. Louis, M. L. Cohen Phys. Rev B 35 (1989) 7840.

[16] K. Kuepper et al., J. Phys.: Condens. Matter 16,8213 (2004).

[17] U. Hiromoto and T. Sakudo, J. Phys. Soc. Jpn. 38, 183 (1975).

[18] J. W. Lui et al., Int. J. Hydrogen Energy 32, 2269 (2007).

[19] M.A. Blanco, E. Francisco and V. Luaña, Comput. Phys. Commun. 158 (2004) 57.

*hamza.tssm@gmaill.com 